\title{
FILTRADO EN LÍNEA DE UNA SEÑAL BASADO EN MÉTODOS ALGEBRAICOS Y SU VALIDACIÓN EXPERIMENTAL
}

\author{
M.P. Portilla ${ }^{1 *}$, J.A. Somolinos ${ }^{1}$, A. López ${ }^{1}$ y R. Morales ${ }^{2}$ \\ ${ }^{1}$ GIT-ERM. Grupo de Investigación Tecnológico en Energías Renovables Marinas \\ DACSON. Escuela Técnica Superior de Ingenieros Navales. UPM. \\ Arco de la Victoria 4. 28040 Madrid. \\ ${ }^{2}$ Escuela Técnica Superior de Ingenieros Industriales. UCLM \\ Campus Universitario s/n.02071 Albacete. \\ \{marinap.portilla, joseandres.somolinos, amable.lopez\}@upm.es, Rafael.morales@uclm.es
}

\begin{abstract}
Resumen
En este trabajo se presenta un nuevo esquema de filtrado en línea de señal basado en el desarrollo algebraico de estimación de parámetros y de estados de reciente introducción. El filtro propuesto se basa en el uso combinado de derivadores temporales algebraicos y una opción de filtrado novedosa. Las principales ventajas del método propuesto son: i) no hay retardos apreciables en la señal filtrada, ii) el método no requiere ningún conocimiento previo de los valores estadísticos del ruido, iii) se obtiene una gran atenuación del ruido, iv) es fácilmente implantable en tiempo-real $y$ v) fácilmente validable en ensayos experimentales. Se presentan resultados experimentales que muestran la bondad del método propuesto.
\end{abstract}

Palabras Clave: Método algebraico, filtrado de señal, medida de profundidad en cuerpos sumergidos.

\section{INTRODUCCIÓN}

Como es ampliamente conocido, en ingeniería y otros campos, las señales medidas son en general, adquiridas por un sistema electrónico a partir de un sensor, un acondicionador electrónico y un sistema de conversión analógico-digital (DAC). Estas señales están inevitablemente corrompidas por ruidos, tales como ruidos de cuantificación en convertidores de baja resolución, acoplamientos eléctricoselectrónicos, etc. Los métodos de filtrado para eliminación del ruido en campos de aplicación software o hardware pueden encontrarse en la literatura [4, 5, 9, 10, 28]. Algunos métodos sencillos de filtrado son los filtros de media móvil, los filtros de suavizado exponencial y de suavizado lineal de Fourier [20]. Una desventaja de estos métodos es la aparición de tiempos de retardo no deseados en las señales filtradas. Otros métodos como los filtros de Wiener o los filtros de Kalman han sido propuestos bajo la hipótesis de que el ruido es un proceso Gaussiano con conocidas propiedades estadísticas [1,
16, 24]. También los filtros basados en métodos frecuenciales pueden causar distorsión en los intervalos de régimen transitorio de la señal con la consiguiente pérdida de información si la señal es noestacionaria [26]. Algunas características de las señales son protegidas con la implementación de filtros más elaborados, tales como los basados en Transformadas Wavelet [2, 17]. Una desventaja de este tipo de filtros es que son relativamente lentos y resultan computacionalmente complejos [3].

Recientemente, se han aplicado técnicas algebraicas par estimación de parámetros y de estado en sistemas lineales con parámetros constantes y coeficientes dependientes del tiempo [13, 21, 27], y también para varios problemas de procesamiento de señal $[15,18]$. Se remarca que los métodos algebraicos no son de naturaleza asintótica y que no requieren del conocimiento estadístico del tipo de ruido que corrompe la señal adquirida (véase $[11,12]$ para más detalles). El ánimo de este trabajo es diseñar un nuevo sistema de filtrado basado únicamente en las técnicas de estimación algebraica de derivadas temporales. La bondad del método propuesto es demostrada en una aplicación de medida de profundidad de un cuerpo sumergido a partir de las medidas de un sensor primario de presión. El algoritmo propuesto utiliza un método algebraico no asintótico para la determinación de las derivadas temporales de la señal corrompida por el ruido y a partir de éstas se obtiene la señal filtrada. Los resultados de ambos estimadores se representan a través de filtros no lineales dependientes del tiempo que permiten estimaciones en línea de las derivadas y de la señal filtrada con una atenuación apreciable en los efectos del ruido. Puesto que la aproximación algebraica de las derivadas es válida únicamente durante un intervalo pequeño de tiempo, se proponen dos líneas temporales que restauran (reset) los intervalos de estimación en periodos regulares de tiempo y descartan los valores no válidos, obteniéndose señales filtradas de muy alta calidad.

Este trabajo está estructurado como sigue: La sección 2 se dedica a la presentación del método algebraico de estimación de derivadas temporales, mientras que 
la sección 3 presenta el método de filtrado propuesto. Las secciones 4 y 5 se dedican a la adaptación de la metodología general a una aplicación de medida de profundidad de un cuerpo sumergido a partir de medidas primarias de presión. La sección 6 está dedicada a la presentación de los resultados experimentales que validan el método propuesto y la sección 7 muestra algunas conclusiones.

\section{CÁLCULO DE DERIVADAS ALGEBRAICAS}

En un sistema observable, el problema de la obtención del estado está íntimamente ligado a la obtención de las sucesivas derivadas de la señal de salida [6]. El método algebraico no asintótico (Ver $[7,8,14])$ se presenta brevemente a continuación.

\subsection{MARCO MATEMÁTICO}

Sea una señal temporal arbitraria suave (smooth) y(t), $\mathrm{y}: \mathbf{R}_{0}^{+} \rightarrow \mathbf{R}$. Ésta puede ser aproximada por su desarrollo en serie de Taylor en torno a un tiempo inicial $\mathrm{t}=\mathrm{t}_{\mathrm{r}}$ como sigue [25]:

$\mathrm{y}(\mathrm{t}) \approx \tilde{\mathrm{y}}(\mathrm{t})=\sum_{\mathrm{i}=1}^{\mathrm{N}} \frac{\mathrm{y}^{(\mathrm{i}-1)}\left(\mathrm{t}_{\mathrm{r}}\right)}{(\mathrm{i}-1) !}\left(\mathrm{t}-\mathrm{t}_{\mathrm{r}}\right)^{\mathrm{i}-1} \cdot \mathrm{H}\left(\mathrm{t}-\mathrm{t}_{\mathrm{r}}\right)$

Donde $\mathrm{H}\left(\mathrm{t}-\mathrm{t}_{\mathrm{r}}\right)$ es la función escalón unitaria de Heaviside y $\mathrm{N}$ representa el orden de truncado de la serie de Taylor. Es posible derivar $\tilde{y}(t)$ al menos $\mathrm{N}$ veces con respecto al tiempo para obtener una expresión idénticamente nula. En el dominio complejo de Laplace, siendo $\tilde{\mathrm{Y}}(\mathrm{s})=\mathrm{L}[\tilde{\mathrm{y}}(\mathrm{t})]$, resulta:

$\left(s^{N} \tilde{Y}(s)-\sum_{i=1}^{N} s^{N-i} y^{(i-1)}\left(t_{r}\right)\right) \cdot e^{-s t_{r}}=0$

La cual conlleva sólo operaciones básicas de cálculo y la expresión $e^{-s t_{r}}$ representa el retardo $t_{r}$. Puesto que es $\mathrm{e}^{-\mathrm{st}_{\mathrm{r}}} \neq 0 \quad \forall \mathrm{t} \geq \mathrm{t}_{\mathrm{r}}$, se obtiene:

$s^{N} \tilde{Y}(s)-\sum_{i=1}^{N} s^{N-i} y^{(i-1)}\left(t_{r}\right)=0$

Con el fin de eliminar las condiciones iniciales, $y^{(i-1)}\left(t_{r}\right)$, es necesario derivar $N$ veces con respecto del operador complejo de Laplace s , obteniéndose:

$\frac{d^{N}}{d s^{N}}\left(s^{N} \tilde{Y}(s)\right)=0$

Por otro lado, la derivada temporal N-sima de $\tilde{y}(t)$ puede expresarse en términos de las derivadas de orden menor. Premultiplicando la expresión (4) por $\mathrm{s}^{-v}$ resulta un sistema recursivo a partir del cual se obtienen todas las derivadas temporales de $\tilde{y}(t)$ : $s^{-v} \frac{d^{N}}{d s^{N}}\left(s^{N} \tilde{Y}(s)\right)=0, v=1,2, \cdots, N-1$

Expresión que contiene información implícita de las derivadas $\mathrm{N}-1$ derivadas de $\mathrm{y}(\mathrm{t})$ en la medida en que aproxima a $\tilde{y}(t)$ según la expresión (1). Según [23] se obtiene el siguiente resultado:

$$
\begin{aligned}
\tilde{y}^{(i)}(t) & =\frac{1}{\left(t-t_{r}\right)^{i}} \frac{(N+i-1) !}{(N-i-1) ! i !} y(t) \\
& +\sum_{j=1}^{i}\left(\prod_{i-j}^{N+i-j-1}\right) \frac{(N-j-1) !}{(N-i-1) !} \frac{z_{j}(N, t)}{\left(t-t_{r}\right)^{N+i-j}}, \quad i=1,2, \cdots, v
\end{aligned}
$$

Donde los estados del filtro $\mathrm{z}_{\mathrm{j}}(\mathrm{N}, \mathrm{t})$, para $\mathrm{j}=1, \cdots, \mathrm{N}-1$ satisfacen:

$\dot{\mathrm{z}}_{\mathrm{j}}(\mathrm{N}, \mathrm{t})=\left(\begin{array}{l}\mathrm{N} \\ \mathrm{j}+1\end{array}\right)^{2}(\mathrm{j}+1) !(-1)^{\mathrm{j}}\left(\mathrm{t}-\mathrm{t}_{\mathrm{r}}\right)^{\mathrm{N}-\mathrm{j}-1} \mathrm{y}(\mathrm{t})+\mathrm{z}_{\mathrm{j}+1}(\mathrm{~N}, \mathrm{t}), \mathrm{j}=1, \cdots, \mathrm{N}-2$

$\dot{\mathrm{Z}}_{\mathrm{N}-1}(\mathrm{~N}, \mathrm{t})=\mathrm{N} !(-1)^{\mathrm{N}-1} \mathrm{y}(\mathrm{t})$

El cual resulta en un filtro lineal de orden $\mathrm{N}-1$, dependiente del tiempo y con condiciones iniciales $\mathrm{z}_{\mathrm{j}}\left(\mathrm{N}, \mathrm{t}_{\mathrm{r}}\right)=0$ para $\mathrm{j}=1, \cdots, \mathrm{N}-1$. Nótese que en $\mathrm{t}=\mathrm{t}_{\mathrm{r}}$ la fórmula anterior genera una indeterminación. De hecho, la precisión finita de cualquier microprocesador numérico, significa que las ecuaciones dadas en (7) no estarán bien definidas durante un tiempo inicial de la forma: $\left[\mathrm{t}_{\mathrm{r}}, \mathrm{t}_{\mathrm{r}}+\varepsilon\right]$. Estas ecuaciones son válidas para $t \geq t_{r}+\varepsilon>0$. Durante el intervalo de tiempo $\left[\mathrm{t}_{\mathrm{r}}, \mathrm{t}_{\mathrm{r}}+\varepsilon\right]$ es posible utilizar un valor arbitrario [22, 23].

\subsection{RESTAURACIÓN Y TÉCNICA DE ESTIMACIÓN POR SOLAPAMIENTO}

La validez de la estimación de $\mathrm{y}^{(\mathrm{i})}(\mathrm{t})$ en el intervalo abierto $\left[\mathrm{t}_{\mathrm{r}}, \mathrm{t}_{\mathrm{r}}+\varepsilon\right)$ se torna cuestionable cuando t aumenta, debido a la naturaleza aproximada de la serie truncada de Taylor en torno a $t_{r}$. Por ello, los cálculos necesitan ser restaurados (reset), y en este trabajo se opta por una restauración a intervalos equidistantes de tiempo $\mathrm{T}_{\mathrm{r}}$. De este modo, el método expuesto ofrece resultados válidos en el intervalo $\left[\mathrm{t}_{\mathrm{r}}+\varepsilon, \mathrm{t}_{\mathrm{r}}+\mathrm{T}_{\mathrm{r}}\right]$, siendo, claramente $\mathrm{T}_{\mathrm{r}} \square \varepsilon$. Las ecuaciones anteriores (1-7) siguen siendo válidas a intervalos de tiempo $t_{r}=k_{r} \cdot T_{r}$, siendo $k_{r} \in N$.

Por otro lado, la no validez de la estimación durante el intervalo $\left[\mathrm{t}_{\mathrm{r}}, \mathrm{t}_{\mathrm{r}}+\varepsilon\right)$ conlleva la siguiente propuesta de definir dos líneas temporales, $t_{1} y$ $\mathrm{t}_{2}$ como sigue (véase la Figura 1). 
$\mathrm{t}_{1}=\mathrm{t} \bmod \mathrm{T}_{\mathrm{r}}$

$\mathrm{t}_{2}=\left(\mathrm{t}-\mathrm{T}_{\mathrm{r}} / 2\right) \bmod \mathrm{T}_{\mathrm{r}}$

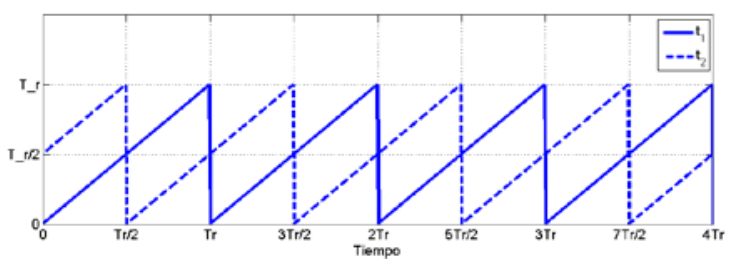

Figura 1: Líneas de tiempo

Para la aplicación propuesta, la estimación de las derivadas $\mathrm{y}_{\mathrm{e}}^{(\mathrm{i})}(\mathrm{t})$ toma la forma:

$\mathrm{y}_{\mathrm{e}}^{(\mathrm{i})}(\mathrm{t})= \begin{cases}\mathrm{y}^{(\mathrm{i})}(\mathrm{t}) & 0 \leq \mathrm{t}<\varepsilon \\ \mathrm{y}_{2 \mathrm{e}}^{(\mathrm{i})}(\mathrm{t}) & 0 \leq\left(\mathrm{t} \bmod \mathrm{T}_{\mathrm{r}}\right)<\mathrm{T}_{\mathrm{r}} / 2 \mathrm{yt}>\varepsilon \\ \mathrm{y}_{1 \mathrm{e}}^{(\mathrm{i})}(\mathrm{t}) & \mathrm{T}_{\mathrm{r}} / 2 \leq\left(\mathrm{t} \bmod \mathrm{T}_{\mathrm{r}}\right)<\mathrm{T}_{\mathrm{r}} \mathrm{yt}>\varepsilon\end{cases}$

\section{FILRADO DE SEÑAL DESDE LA PERSPECTIVA ALGEBRAICA}

Sea una señal suave (smooth) $\mathrm{x}(\mathrm{t})$, afectada por un ruido aditivo $\mu(\mathrm{t})$ de naturaleza desconocida que proporciona una señal medida $y(t)$ :

$y(t)=x(t)+\mu(t)$

No resulta conveniente el uso de la señal anterior para implementar un sistema de control por realimentación que requiera derivadas, debido a los efectos de ruido. La alternativa a usar filtros pasobajo es ampliamente conocida (ver [19] por ejemplo). En cualquier caso, el uso de filtros produce retardos en la señal filtrada. De un modo aproximado, cuanto menor es la frecuencia de corte y mayor el orden del filtro, mayor es el retardo provocado en la señal filtrada. Otras técnicas como los observadores de Luemberger ofrecen una convergencia asintótica bajo ciertas condiciones que requieren el conocimiento del sistema, o los filtros de Kalman que requieren del conocimiento de las propiedades estadísticas de la señal a filtrar. El objetivo principal del filtrado algebraico es enunciado como sigue:

Dada una señal ruidosa $\mathrm{y}(\mathrm{t})$, definida por la expresión (10), diseñar un filtro algebraico que reconstruya la señal $\mathrm{x}(\mathrm{t}) \sin$ retardos.

\subsection{MARCO MATEMÁTICO}

En las vecindades de $t=t_{r f}$, una aproximación de la derivada temporal j-ésima de la señal es de la forma:

$\mathrm{y}^{(\mathrm{j})}(\mathrm{t}) \cdot \mathrm{H}\left(\mathrm{t}-\mathrm{t}_{\mathrm{rf}}\right)=\mathrm{y}_{\mathrm{e}}^{(\mathrm{j})}(\mathrm{t}) \cdot \mathrm{H}\left(\mathrm{t}-\mathrm{t}_{\mathrm{rf}}\right)$
Donde $\mathrm{H}\left(\mathrm{t}-\mathrm{t}_{\mathrm{rf}}\right)$ es la función escalón unitaria de Heaviside e $_{\mathrm{e}}^{(\mathrm{j})}(\mathrm{t})$ denota la derivada temporal $\mathrm{j}$ ésima de la señal estimada $y_{e}(t)$. Se propone el filtrado algebraico mediante el siguiente procedimiento:

1.- Obtener las Transformadas de Laplace de las expresiones anteriores $\mathrm{y}$ definir la variable $\Phi(\mathrm{s})=\mathrm{L}\left[\mathrm{y}_{\mathrm{e}}^{(\mathrm{j})}(\mathrm{t})\right]:$

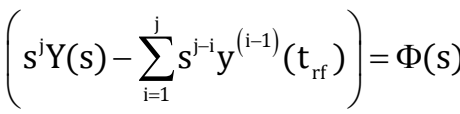

2.- Diferenciar $\mathrm{j}$ veces con respecto al operador complejo de Laplace $\mathrm{s}$, con el fin de eliminar las condiciones iniciales $\mathrm{y}^{(\mathrm{i}-1)}\left(\mathrm{t}_{\mathrm{rf}}\right)$.

3.- Multiplicar por $\mathrm{s}^{-j}$ para eliminar los términos de derivadas caracterizados por potencias positivas de s y manipular la expresión resultante para obtener el término $\mathrm{d}^{\mathrm{j}} \mathrm{Y}(\mathrm{s}) / \mathrm{d} \mathrm{s}^{\mathrm{j}}$.

4.- Trasladar la expresión obtenida al dominio temporal de acuerdo con las reglas usuales de la Transformada Inversa de Laplace y obtener la señal filtrada $\mathrm{y}_{\mathrm{e}}(\mathrm{t}) \approx \mathrm{x}(\mathrm{t})$.

\subsection{RESTAURACIÓN Y TÉCNICA DE ESTIMACIÓN POR SOLAPAMIENTO}

De un modo similar al llevado a cabo en el apartado 2 , se toman dos líneas de tiempo $t_{\mathrm{f} 1} \mathrm{y}_{\mathrm{f} 2}$ para evitar las singularidades a cada comienzo de intervalos de tiempo equidistantes, en este caso con intervalo $\mathrm{T}_{\mathrm{rf}}$, y para garantizar que la estimación de la señal truncada por serie de Taylor no se desvíe de la deseada al incrementar el tiempo t, se toma un intervalo $\mathrm{T}_{\mathrm{rf}} \square \varepsilon$, los tiempos de reset $\mathrm{t}_{\mathrm{rf}}=\mathrm{k}_{\mathrm{rf}} \cdot \mathrm{T}_{\mathrm{rf}}$ siendo, también en este caso, $\mathrm{k}_{\mathrm{rf}} \in \mathrm{N}$. Así pues, de un modo similar al utilizado en la ecuación (8), las dos líneas de tiempo vienen determinadas por:

$\mathrm{t}_{1 \mathrm{f}}=\mathrm{t} \bmod \mathrm{T}_{\mathrm{rf}}$

$\mathrm{t}_{2 \mathrm{f}}=\left(\mathrm{t}-\mathrm{T}_{\mathrm{rf}} / 2\right) \bmod \mathrm{T}_{\mathrm{rf}}$

El resultado de estas dos líneas de tiempo es similar al mostrado en la figura 1 .

\section{APLICACIÓN A LA MEDIDA DE PROFUNDIDAD DE UN CUERPO SUMERGIDO}

Para el caso a analizar en este trabajo se han particularizado las ecuaciones (1-4) a $\mathrm{N}=7 \mathrm{y}$ a la obtención de las 3 primeras derivadas. De esta forma, 
las expresiones indicadas en la ecuación (7) para los intervalos $\mathrm{t} \in\left[\mathrm{T}_{\mathrm{r}}, \mathrm{T}_{\mathrm{r}}+\varepsilon\right)$ y $\mathrm{t} \in\left[\mathrm{T}_{\mathrm{r}}+\varepsilon, 2 \mathrm{~T}_{\mathrm{r}}\right)$ son:

$$
\begin{aligned}
& \dot{y}_{e}(t)=\left\{\begin{array}{cr}
\dot{y}(t) & t \in\left[T_{r}, T_{r}+\varepsilon\right. \\
\frac{\left[42\left(t-T_{r}\right)^{6} y(t)+z_{1}(t)\right]}{\left(t-T_{r}\right)^{7}} & t \geq T_{r}+\varepsilon
\end{array}\right. \\
& \ddot{y}_{e}(t)=\left\{\begin{array}{c}
\ddot{y}(t) \\
\frac{\left[840\left(t-T_{r}\right)^{6} y(t)+35 z_{1}(t)+\left(t-T_{r}\right) z_{2}(t)\right]}{\left(t-T_{r}\right)^{8}}
\end{array}\right. \\
& \dddot{y}(t) \\
& \dddot{y}_{e}(t)=\left\{\begin{array}{l}
{\left[\begin{array}{l}
10080\left(t-T_{r}\right)^{6} y(t)+560 z_{1}(t)+ \\
+28\left(t-T_{r}\right) z_{2}(t)+\left(t-T_{r}\right)^{2} z_{3}(t)
\end{array}\right]} \\
\left(t-T_{r}\right)^{9}
\end{array}\right.
\end{aligned}
$$

Donde

$$
\begin{aligned}
& \dot{\mathrm{z}}_{1}(\mathrm{t})=-882\left(\mathrm{t}-\mathrm{T}_{\mathrm{r}}\right)^{5} \mathrm{y}(\mathrm{t})+\mathrm{z}_{2}(\mathrm{t}) \\
& \dot{\mathrm{z}}_{2}(\mathrm{t})=7350\left(\mathrm{t}-\mathrm{T}_{\mathrm{r}}\right)^{4} \mathrm{y}(\mathrm{t})+\mathrm{z}_{3}(\mathrm{t}) \\
& \dot{\mathrm{z}}_{3}(\mathrm{t})=-29400\left(\mathrm{t}-\mathrm{T}_{\mathrm{r}}\right)^{3} \mathrm{y}(\mathrm{t})+\mathrm{z}_{4}(\mathrm{t}) \\
& \dot{\mathrm{z}}_{4}(\mathrm{t})=52920\left(\mathrm{t}-\mathrm{T}_{\mathrm{r}}\right)^{2} \mathrm{y}(\mathrm{t})+\mathrm{z}_{5}(\mathrm{t}) \\
& \dot{\mathrm{z}}_{5}(\mathrm{t})=-35280\left(\mathrm{t}-\mathrm{T}_{\mathrm{r}}\right) \mathrm{y}(\mathrm{t})+\mathrm{z}_{6}(\mathrm{t}) \\
& \dot{\mathrm{z}}_{6}(\mathrm{t})=5040 \mathrm{y}(\mathrm{t})
\end{aligned}
$$

En cuanto al filtrado propiamente dicho, se toma el orden del filtro $j=3$. Los pasos indicados en la sección 3.1. son ahora, particularizados:

Paso 1: recuérdese que $\Phi(\mathrm{s})=\mathrm{L}\left[\dddot{\mathrm{y}}_{\mathrm{e}}(\mathrm{t})\right]$ )

$$
s^{3} Y(s)-s^{2} y\left(T_{r f}\right)-s \dot{y}\left(T_{r f}\right)-\ddot{y}\left(T_{r f}\right)=\Phi(s)
$$

Paso 2: se deriva tres veces con respecto al operador complejo de Laplace $s$. De esta forma se eliminan las tres condiciones iniciales: $\mathrm{y}\left(\mathrm{T}_{\mathrm{rf}}\right), \dot{\mathrm{y}}\left(\mathrm{T}_{\mathrm{rf}}\right)$ e $\ddot{\mathrm{y}}\left(\mathrm{T}_{\mathrm{rf}}\right)$

$$
6 \mathrm{Y}(\mathrm{s})+18 \mathrm{~s} \frac{\mathrm{dY}(\mathrm{s})}{\mathrm{ds}}+9 \mathrm{~s}^{2} \frac{\mathrm{d}^{2} \mathrm{Y}(\mathrm{s})}{\mathrm{ds^{2 }}}+\mathrm{s}^{3} \frac{\mathrm{d}^{3} \mathrm{Y}(\mathrm{s})}{d \mathrm{~s}^{3}}=\frac{\mathrm{d}^{3} \Phi(\mathrm{s})}{\mathrm{ds^{3 }}}
$$

Paso 3: se divide por $s^{3}$ para eliminar los términos caracterizados por potencias positivas de $\mathrm{s}$.

$\frac{d^{3} Y(s)}{d s^{3}}=\frac{d^{3} \Phi(s)}{d s^{3}}-6 s^{-3} Y(s)+18 s^{-2} \frac{d Y(s)}{d s}+9 s^{-1} \frac{d^{2} Y(s)}{d s^{2}}$

Paso 4: se obtiene la expresión dada en la ecuación (18) al dominio temporal, teniendo en cuenta que en las vecindades de $t=T_{r f}$ es aplicable la propiedad de la Transformada inversa de Laplace siguiente $L^{-1}\left[\frac{d^{j} Y(s)}{d s^{j}}\right]=(-1)^{j}\left(t-T_{r f}\right)^{j} y(t)$

$\mathrm{y}_{\mathrm{e}}(\mathrm{t})=\frac{\int_{\mathrm{T}_{\mathrm{ff}}}^{\mathrm{t}} \int_{0}^{\tau_{1}} \int_{0}^{\tau_{2}}\left(\tau_{3}^{3} \dddot{y}_{\mathrm{e}}\left(\tau_{3}\right)+6 \mathrm{y}\left(\tau_{3}\right)\right) \mathrm{d} \tau_{3} \mathrm{~d} \tau_{2} \mathrm{~d} \tau_{1}-18 \int_{T_{\mathrm{rf}}}^{\mathrm{t}} \int_{0}^{\tau_{1}}\left(\tau_{2} \mathrm{y}\left(\tau_{2}\right)\right) \mathrm{d} \tau_{2} \mathrm{~d} \tau_{1}+9 \int_{T_{\mathrm{rf}}}^{\mathrm{t}}\left(\tau_{1}^{2} \mathrm{y}\left(\tau_{1}\right)\right) \mathrm{d} \tau_{1}}{\left(\mathrm{t}-\mathrm{T}_{\mathrm{rf}}\right)^{3}}$

De un modo similar al llevado a cabo para la estimación de las derivadas algebraicas (ecuación (9)), la señal estimada resultado de filtrar $y(t)$ definida en la ecuación (10) resulta:

$$
\mathrm{x}_{\mathrm{e}}(\mathrm{t})=\left\{\begin{array}{cc}
\mathrm{y}(\mathrm{t}) & 0 \leq \mathrm{t}<\varepsilon \\
\mathrm{y}_{2 \mathrm{e}}(\mathrm{t}) & 0 \leq\left(\mathrm{t} \bmod \mathrm{T}_{\mathrm{rf}}\right)<\mathrm{T}_{\mathrm{rf}} / 2 \mathrm{yt}>\varepsilon \\
\mathrm{y}_{1 \mathrm{e}}(\mathrm{t}) & \mathrm{T}_{\mathrm{rf}} / 2 \leq\left(\mathrm{t} \bmod \mathrm{T}_{\mathrm{rf}}\right)<\mathrm{T}_{\mathrm{rf}} \mathrm{yt}>\varepsilon
\end{array}\right.
$$

\section{FILTRADO DE SEÑAL EN LÍNEA}

En esta sección, se describe el esquema final de filtrado de señal en línea, basado en los métodos algebraicos descritos en las secciones 2 y 3 , y particularizado para este caso en la sección 4. El algoritmo propuesto se lleva a cabo en dos etapas como se muestra en la figura 2. La primera etapa consiste en un estimador de derivadas algebraicas a partir de la señal medida $\mathrm{y}(\mathrm{t})$, basado en el solapamiento de las estimaciones algebraicas para cada una de las mitades de las líneas de tiempo $\mathrm{t}_{1} \mathrm{y}$ $\mathrm{t}_{2}$ a excepción del intervalo inicial $[0, \varepsilon)$ donde se toman las derivadas directas de la señal ruidosa, fuertemente deterioradas por la presencia de ruido. El estimador de derivadas ofrece las tres primeras derivadas a partir de las ecuaciones (14) y (15). En la segunda etapa, se obtiene la señal filtrada a partir de la señal ruidosa $y(t)$ y la tercera derivada estimada $\dddot{y}_{\mathrm{e}}(\mathrm{t})$, también basado en la técnica de solapamiento que permite obtener $\mathrm{x}_{\mathrm{e}}(\mathrm{t})$ de acuerdo con las ecuaciones (19-20). La señal filtrada obtenida tiene las siguientes propiedades: i) alta robustez ante ruido, ii) robustez intrínseca independientemente de las propiedades estadísticas del ruido, iii) fácil implementación y alta versatilidad y iv) puede llevarse a cabo el filtrado en tiempo real y en línea. La siguiente sección muestra los resultados experimentales de validación llevados a cabo en el Laboratorio. 


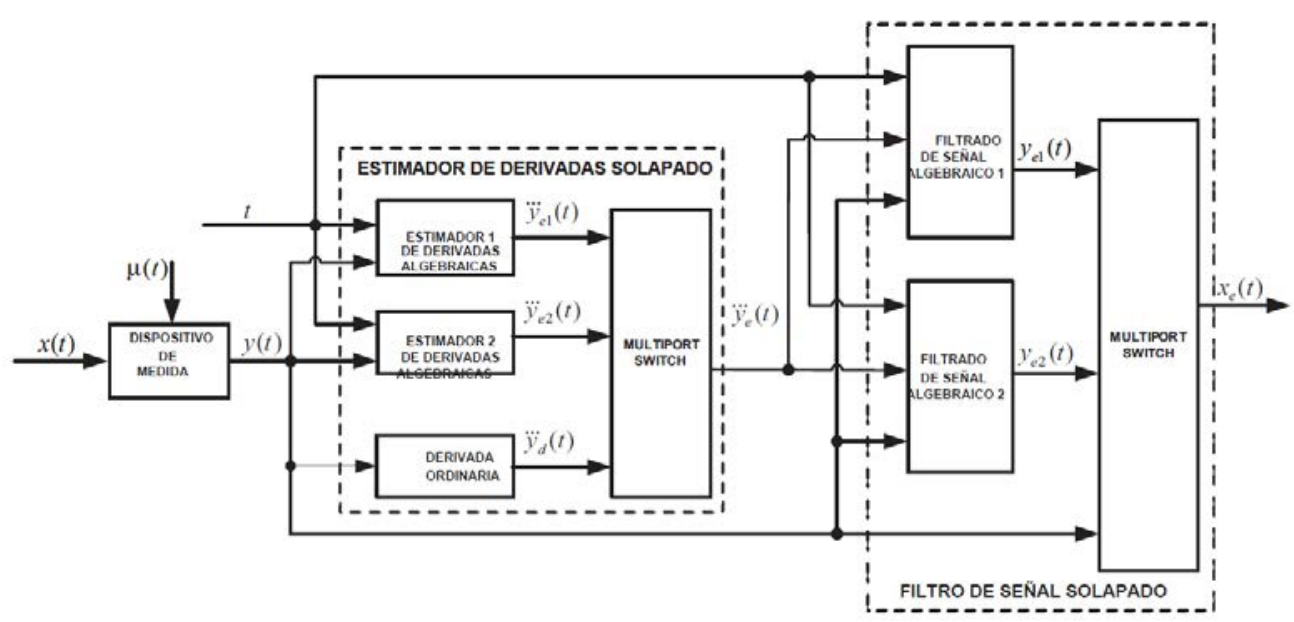

Figura 2. Esquema del filtro algebraico propuesto

\section{6 \\ RESULTADOS EXPERIMENTALES}

El filtro propuesto ha sido validado llevando a cabo experimentos basados en mediciones de la profundidad de columna de agua de un cuerpo sumergido a partir de las medidas de un sensor de presión. Se denota a esta medida "profundidad". El sensor primario convierte la presión en una deformación de membrana que es convertida a una tensión eléctrica mediante galgas extensiométricas y un puente de Wheatstone. Después de una breve descripción del entorno de desarrollo, se presentan los resultados experimentales.

\subsection{ENTORNO DE EXPERIMENTACIÓN}

Un sensor DPF E13-VF con un fondo de escala de hasta 1.6 bares se ha utilizado como sensor primario. Éste proporciona una salida de 0 a $10 \mathrm{~V}$ con un tiempo de subida (10-90\%) menor a $1 \mathrm{~ms}$ y pequeños errores $\left( \pm 0.5\right.$ de fondo de escala a $20^{\circ} \mathrm{C}$ ). Un cable apantallado de tres hilos se utiliza para alimentar al sensor $\left(24 \mathrm{~V}_{\mathrm{DC}}\right)$ y para transmitir el voltaje analógico desde el sensor al sistema de adquisición de datos. La malla eléctrica que recubre al cable se conecta con el agua donde se sumerge el sensor, y que actúa como masa y nodo de referencia de tensión nula.

El sensor fue ubicado en el extremo de una barra metálica de $2 \mathrm{~m}$ que está dividida en pasos fijos de 1 $\mathrm{cm}$. El sensor está dispuesto de forma perpendicular a la barra. La barra fue pintada de color amarillo para un buen contraste óptico con la oscuridad del Canal de Ensayos Hidrodinámicos de la ETS de Ingenieros Navales de la Universidad Politécnica de Madrid, y cuya profundidad es de hasta $2.2 \mathrm{~m}$.

Se implementaron dos sistemas independientes de adquisición de datos. El primero utiliza un osciloscopio Tektronix DPO-4034 con sondas pasivas P2200, 1X. La salida del sensor fue conectada al canal 1 de este osciloscopio sin conexión a masa. Una señal de sincronismo de $0-5$ $\mathrm{V}$ fue utilizada como señal de disparo en el canal 2. Una vez que esta señal de disparo se ha recibido, todas las medidas son adquiridas mediante un PC con el Instrumentation Toolbox de MATLAB y una conexión USB bajo el estándar VISA con el osciloscopio. El osciloscopio ofrece un convertidor Analógico-Digital de un único byte con un rango entero de -128 a 127.

El segundo sistema utiliza una tarjeta National Instruments PCI-6221 tanto para la adquisición del voltaje analógico del sensor como para generar la señal de disparo mencionada anteriormente. Utiliza un convertidor Analógico-Digital de 16 bits y se ha utilizado una conexión diferencial y apantallada con el sensor. Se ha utilizado el entorno de MATLABSimulink con el Real-Time Workshop para las medidas de profundidad y la implementación del filtro propuesto. La figura 3 muestra el esquema de integración de este segundo sistema.

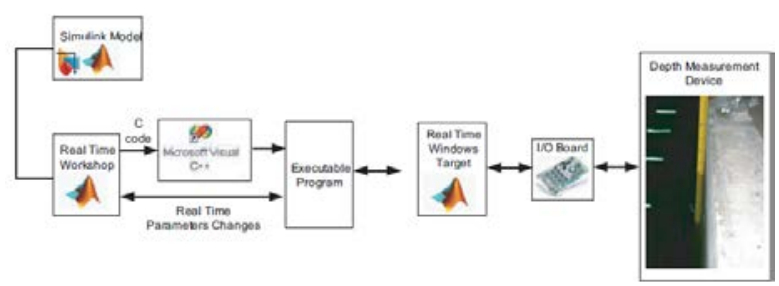

Figura 3. Esquema de medida con el filtro propuesto

Finalmente, la figura 4 muestra una vista general del entorno de experimentación con los dos sistemas de adquisición descritos anteriormente y ambas interfaces gráficas. Puede apreciarse en la fotografía el osciloscopio con las dos sondas de medida, así como la pantalla del computador durante uno de los experimentos, junto con la barra amarilla en la parte derecha de la misma. El canal de ensayos está a la espalda del conjunto. 


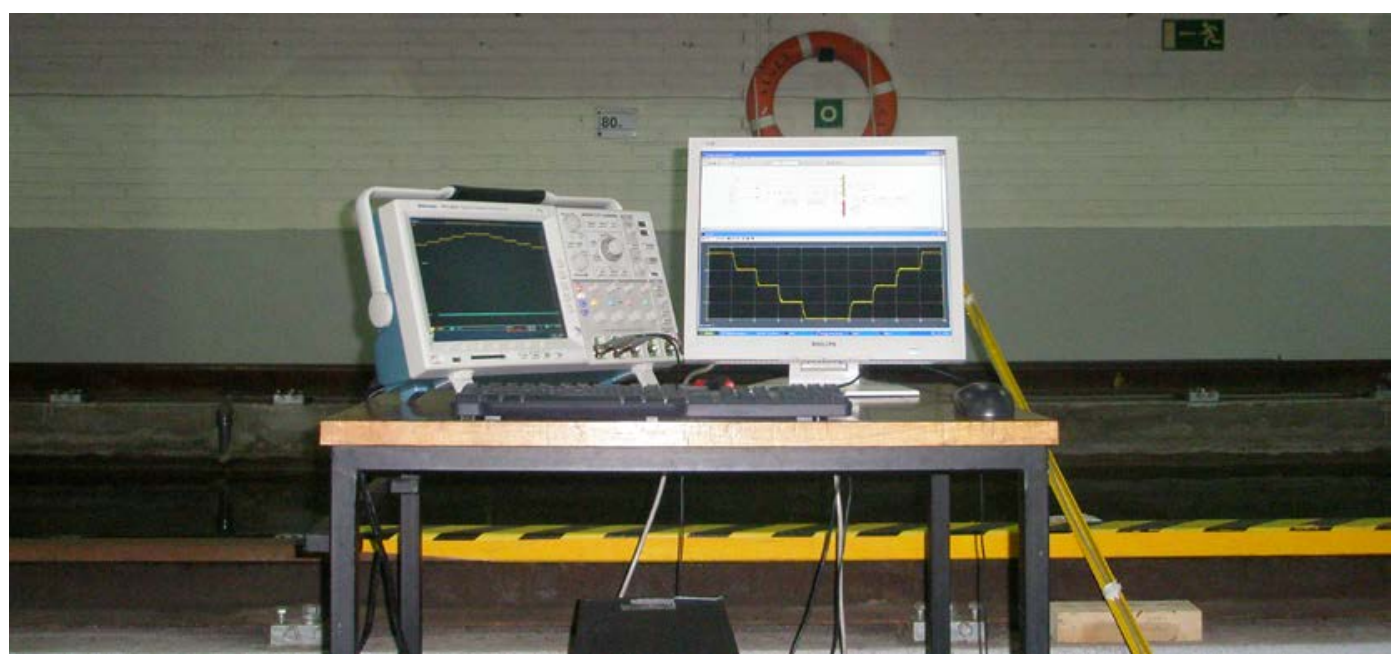

Figura 4. Entorno de Experimentación

\subsection{RESULTADOS EXPERIMENTALES}

La figura 5 muestra la señal obtenida con un ADC de 8 bits del osciloscopio (color azul) junto con la señal filtrada $\mathrm{x}_{\mathrm{e}}(\mathrm{t})$ en rojo. Se han utilizado unos tiempos $\mathrm{T}_{\mathrm{r}}=8 \mathrm{~s}$ y $\mathrm{T}_{\mathrm{rf}}=0.5 \mathrm{~s}$ con periodos de muestreo de la señal a medir de $10 \mathrm{~ms}$.

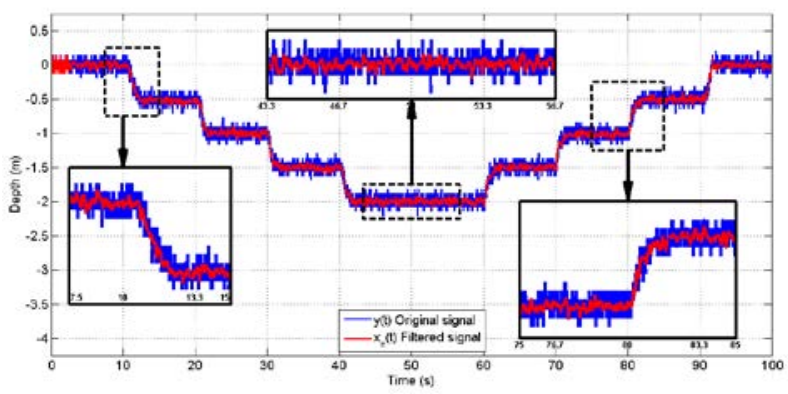

Figura 5. Señal original (ADC 8 bits) y señal filtrada

La figura 6 siguiente muestra tres juegos de la misma señal anterior: La señal filtrada con el filtro propuesto (rojo), la señal filtrada con un filtro paso bajo de Butterworth de orden 12 y frecuencia de corte relativa de 0.05 (magenta) y la señal obtenida con el sistema ADC de 16 bits (negro).

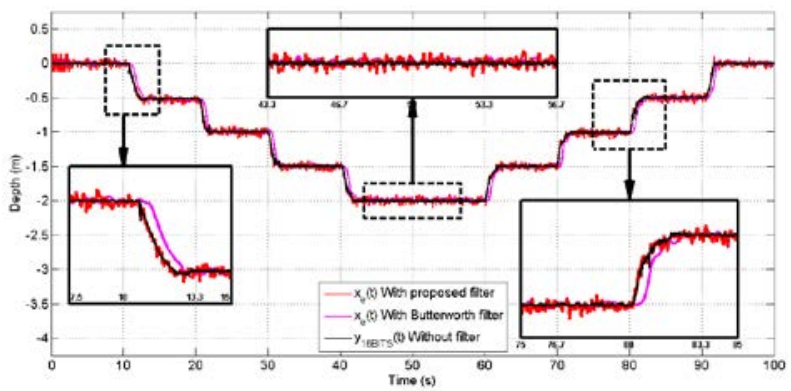

Figura 6. Señal filtrada y señal original (ADC 16 bits).
Puede comprobarse el retardo que ofrece el filtro de Butterworth a la vez que se comprueba la bondad de la señal filtrada obtenida en lo que se refiere a la ausencia de retardos por comparación con la señal medida a partir del ADC de 16 bits diferencial y apantallado que no ofrece ruido apreciable.

Las figura 7 y 8 muestran los mismos dos juegos de señales para mediciones dinámicas en otro ensayo.

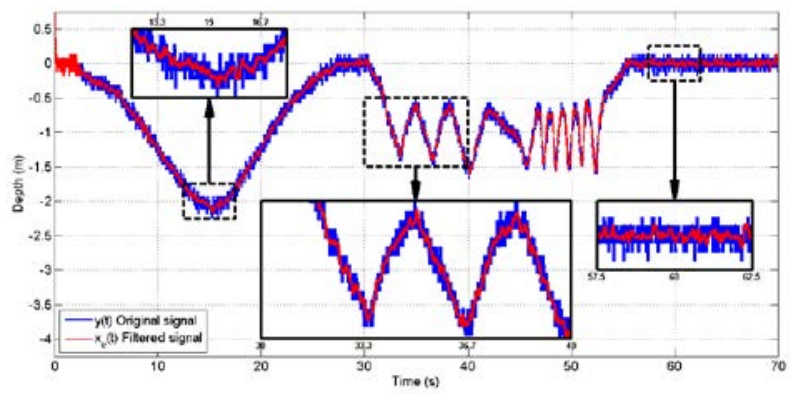

Figura 7. Señal original (ADC 8 bits) y señal filtrada

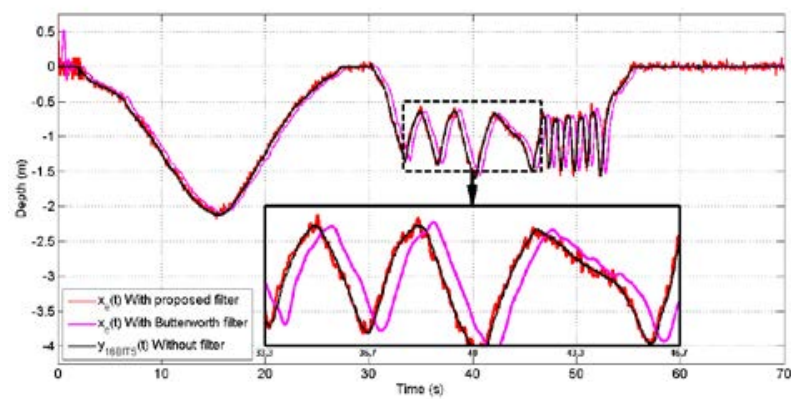

Figura 8. Comparación entre señales.

Finalmente, la figura 9 muestra la estimación de la primera derivada para este segundo ensayo en comparación con la derivada directa de la señal a partir de las medidas del ADC de 8 bits y con la derivada de la señal filtrada obtenida. 


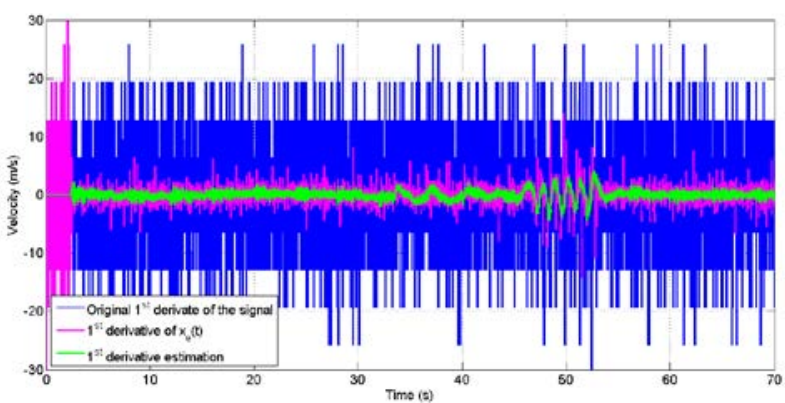

Figura 9. Comparación de velocidades obtenidas del segundo ensayo.

\section{CONCLUSIONES}

Se ha presentado un nuevo algoritmo de filtrado para señales afectadas por ruido aditivo. El filtro propuesto está basado en las técnicas de estimación algebraica de parámetros y de derivadas temporales. El filtro propuesto se compone de dos subsistemas: i) el primero es una implementación solapada de un estimador algebraico de derivadas y ii) una implementación solapada del filtro propiamente dicho. El subsistema de estimación de derivadas algebraicas está basado en estimadores conmutados que obtienen cada una de las señales requeridas concatenando las estimaciones en intervalos de tiempo en los que la aproximación truncada por serie de Taylor no diverge, y en los que no se producen singularidades en los instantes iniciales de cada intervalo de estimación. La misma política de reset y doble estimador se ha utilizado para obtener la señal filtrada. Se ha validado experimentalmente la bondad del algoritmo propuesto con respecto a diferentes propiedades de robustez de la estimación. Las siguientes conclusiones merecen ser resaltadas: i) No hay aparente retardo en la señal filtrada, ii) alta robustez de la señal en lo que se refiere a desconocimento de las propiedades estadísticas del ruido, iii) alta atenuación del ruido, iv) el algoritmo puede implementarse en tiempo real y en línea y v) su programación resulta muy intuitiva y sencilla, habida cuenta de que se trata de computar polinomios de potencias del tiempo transcurrido para cada intervalo de estimación. El algoritmo ha sido utilizado para obtener medidas de profundidad de un cuerpo sumergido y sus dos primeras derivadas.

\section{Agradecimientos}

Este trabajo ha sido financiado parcialmente por el Ministerio de Economía y Competitividad bajo la concesión DPI2014-53499R. Los autores quieren agradecer la participación del personal del Canal de Ensayos de la ETS I Navales de la Universidad Politécnica de Madrid en la realización de los ensayos.

\section{Referencias}

[1] A. Alessandri (2000). Design of sliding-mode observers and filters for nonlinear dynamic systems, IEEE 39th Conference on Decision and Control, vol. 3(11), pp. 2593-2598.

[2] M. Alfaouri, K. Daqrouq (2008). ECG denoising by sparse wavelet shrinkage, American Journal of Applied Sciences, vol. 5(3), pp. 276-281.

[3] J. Cortés Romero, C. García-Rodríguez, A. Luviano-Juárez, R. Portillo-Vélez and H. SiraRamírez (2009). An algebraic denoising scheme, $6^{\text {th }}$ International Conference on Electrical Engineering, Computing Science and Automatic Control, pp. 1-5.

[4] R.M. Crozier, B.M.G. Cheetham, C. Holt and E. Munday (1993). Speech Enhancement Employing Spectras subtraction and Linear Predictive Analysis, IEEE Electronic Letters, pp. 1094-1095.

[5] G. Devuyst, J.M. Vesin, P.A. Despland and J. Bogousslavsky (2000). The matching pursuit: $a$ new method of characterizing microembolic signals, Ultrasound in Med. \& Biol., pp. 10511056.

[6] S. Diop and M. Fliess (1991). Nonlinear observability, identifiability and persistent trajectories, Proceedings of the 36th IEEE Conference on Decision and Control, Brighton, England, 1991, pp. 714-719.

[7] S. Diop, J.W. Grizzle and F. Chaplais (2000). On numerical differentiation algorithms for nonlinear estimation, Proceedings of the 39th IEEE Conference on Decision and Control, Sydney, Australia, 2000, pp. 1133-1138.

[8] S. Diop, J.W. Grizzle, F. Chaplais and A. Stefanopoulou (1994). Interpolation and numerical differentiation for observer design, Proceedings of the American Control Conference, Baltimore, USA, 1994, pp. 13291333.

[9] D.L. Donoho (1995). De-noising by softthresholding, IEEE Transactions on Information Theory, pp. 613-627.

[10] Y. Ephaim and D. Malah (1984). Speech enhancement using a minimum mean-square error short-time spectral amplitude estimator, IEEE Transactions Acoustic, Speech and Signal Processing, pp. 1109-1121. 
[11] M. Fliess (2006). Analyse non standard du bruit, C.R. Acad. Sci. Paris, p. Ser. I 342.

[12] M. Fliess (2008). Critique du rapport signal bruit en communications numèriques Questioning the signal to noise ratio in digital communications, International Conference in Honor of Claude Lobry, ARIMA (Revue africaine d'informatique et the Mathmatiques appliques) vol. 9, pp. 419-429.

[13] M. Fliess and H. Sira-Ramírez (2003). An algebraic framework for linear identification, Optimization and Calculus of Variations, vol. 9, pp. 151-168.

[14] A. Levant (1998). Robust exact differentiation via sliding mode technique, Automatica, vol. 34(3), pp. 379-384.

[15] D.Y Liu, O. Gibaru and W. Perruquetti (2011). Differentiation by integration with Jacoby polynomials, J. Comput. Appl. Math, vol. 235, pp.3015-3032.

[16] G. Lu, et.al. (2009). Removing ECG noise from surface EMG signals using adaptive filtering, Neuroscence Letters, vol.462(1), pp. 14-19.

[17] S. Mallat (1999). A Wavelet tour of signal processing, Academic Press, San Diego, CA, USA. 1999

[18] M. Mboup (2009). Parameter estimation for signals described by differential equations, Applicable Analysis, vol. 88, 29-52.

[19] K. Ogata (2010). Modern Control Engineering (5th Edition). Prentice Hall.

[20] J.G Proakis and D.G. Manolakis (2006). Digital Signal Processing - Principles, Algorithms and Applications, 4th Edition, Pearson.
[21] R. Morales, H. Sira-Ramírez and V. Feliu (2011). Nonlinear control for magnetic levitation systems based on fast online algebraic identification of the input gain, IEEE Transactions on Control Systems Technology, vol. 19(4), pp. 757-771.

[22] R. Morales, J. Somolinos and H. Sira-Ramírez (2014). Control of a DC motor using algebraic derivative estimation with real time experiments. Measurement, vol. 47, pp. 401417.

[23] J. Reger, H. Sira-Ramírez and M. Fliess (2005). On-non-asymptotic state estimation of nonlinear systems, Proc. 44th IEEE Conference on Decision and Control (CDC'05), Seville.

[24] O. Sayadi and M.B. Shamsollahi (2008). ECG denoising and compression using a modified extended Kalman filter structure, IEEE Transactions on Biomedical Engineering, vol. 55(9), pp. 2240-2248.

[25] H. Sira-Ramírez, C. García-Rodríguez, J. Cortés-Romero and A. Luviano-Juárez, Algebraic Identification and Estimation Methods in Feedback Control Systems, in: Wiley, Chicester, West Sussex, United Kingdom, ISBN 9781118730607, 2014.

[26] M. Üntündag, A. Sengür, M. Gökbulut and F. Ata (2013). Performance comparison of wavelet thresholding techniques on weak EGC signal denoising, Przeglad Elektrotechniczny, ISSN 0033-2097, R. 89 NR 5/2013.

[27] W. Yang Tina, T. Floquet and W. Perruquetti (2008). Fast state estimation in linear timevarying systems: An algebraic approach, Proceedings of the 47th IEEE Conference on Decision and Control, pp. 2539-2544.

[28] L. Yue and B. Yang (2004). Introduction of chaotic oscillator detection, Beijing: Publish House of Electronics Industry. 